\title{
Adverse effects of isoniazid preventative therapy for latent tuberculosis infection: a prospective cohort study
}

Justin T Denholm ${ }^{1,4,5}$

Emma S McBryde ${ }^{1,3}$

Damon P Eisen ${ }^{1,3}$

Jocelyn S Penington'

Caroline Chen ${ }^{2}$

Alan C Street ${ }^{1}$

'Victorian Infectious Diseases Service, ${ }^{2}$ Department of Pharmacy, Royal Melbourne Hospital, Parkville, VIC, Australia; ${ }^{3}$ Department of Medicine (RMH/WH), ${ }^{4}$ Department of Microbiology and Immunology, University of Melbourne, Parkville, VIC, Australia; ${ }^{5}$ Victorian Tuberculosis Program, Melbourne Health, Parkville, VIC, Australia

Correspondence: Justin T Denholm Victorian Infectious Diseases Service, Royal Melbourne Hospital, Parkville, VIC 3050, Australia

Tel +6I 393427000

Fax +6I 393427277

Email justin.denholm@mh.org.au
This article was published in the following Dove Press journal:

Drug, Healthcare and Patient Safety

20 October 2014

Number of times this article has been viewed

Introduction: Isoniazid preventative therapy (IPT) is a widely used intervention for treatment of latent tuberculosis infection (LTBI), particularly in patients at high risk for reactivation. While treatment-limiting adverse effects have been well studied, few prospective studies have considered the range of adverse effects that patients may experience with IPT.

Methods: All patients commencing treatment for LTBI were prospectively enrolled in an ongoing database of LTBI treatment outcomes particularly related to adverse effects, treatment adherence, and treatment completion.

Results: Data on the first 100 patients who were prescribed IPT are presented. Fifty-six patients reported at least one adverse effect at some stage during treatment, with six experiencing at least one World Health Organization (WHO) Grade 3-4 adverse effect. Increased age was significantly associated with risk of adverse effects (odds ratio $[\mathrm{OR}]=1.05$ per year; confidence interval $[\mathrm{CI}]$ of $1.02-1.08=95 \%$ ). Eighty-five patients had documented completion of therapy locally, with ten patients ceasing IPT due to adverse effects.

Discussion: This report highlights a variety of somatic adverse effects that occurred in a realworld cohort of patients receiving IPT. While adverse effects were frequently identified in this study, the considerable majority were low grade and transient. Despite frequent adverse effects of LTBI in our treatment cohort, the study demonstrated high levels of treatment adherence and completion.

Keywords: latent tuberculosis infection, isoniazid preventative therapy, treatment toxicity

\section{Introduction}

Isoniazid preventative therapy (IPT) is a widely-used intervention for treatment of latent tuberculosis infection (LTBI). Most commonly consisting of a 6- to 9-month course of daily isoniazid, the use of treatment to prevent tuberculosis infection has previously been focused on household contacts of those with active disease. ${ }^{1}$ However, in recent years, an expanding group of patients has been recognized as being at higher risk of LTBI reactivation. Patients within this group are receiving immunosuppressive medications, especially tumor necrosis factor alpha (TNF- $\alpha$ ) inhibitors, and are routinely recommended IPT prior to the initiation of therapy. ${ }^{2}$

Given the increasing number of patients with significant comorbidities likely to receive IPT in the future, it is important to assess the impact of therapy in real-world cohorts, particularly in relation to adverse effects and treatment completion. Previous reports have tended to emphasize serious treatment limiting adverse effects, particularly hepatotoxicity, which has been well-studied in prospective cohorts. ${ }^{3}$ However, a wide variety of less well-documented adverse effects have been observed in association 
with IPT, including acneiform and other rashes, gastrointestinal adverse effects, peripheral neuropathy, drowsiness, and alopecia, many of which have been reported in case reports or series but not prospectively considered in a real-world cohort. $^{4-7}$ The frequency with which many of these adverse effects occur is poorly defined, yet may have substantial impact on patient willingness to initiate or complete therapy, particularly as patients with LTBI are not symptomatic. We therefore aimed to prospectively study the adverse effects of IPT in a real-world cohort, and consider their frequency, severity and association with failure to complete therapy.

\section{Methods}

This study was approved by the Melbourne Health Human Research Ethics Committee as a quality assurance activity. The requirement for participant consent was waived, as all information was collected as part of routine clinical care. From June 2011, all patients commencing treatment for LTBI at the Royal Melbourne Hospital were prospectively and sequentially enrolled in an ongoing database of LTBI treatment outcomes, particularly related to adverse effects and treatment adherence. Routine clinical review was conducted via outpatient clinics at $0,1,3,6$, and 9 months for those receiving 9-month courses of $300 \mathrm{mg}$ daily IPT, with liver function testing performed prior to commencement but not routinely thereafter, except for patients with existing liver disease or baseline liver function abnormalities. ${ }^{8}$ At each visit, medication was dispensed for patient self-administration, without direct observation. Pyridoxine supplementation was provided routinely for patients considered to be at risk of malnutrition. Patients were advised to abstain from alcohol. At the time of each visit, a standardized reporting form was completed by clinicians who recorded treatment adherence and the presence and severity of any adverse effects during the preceding period of therapy. Any adverse event was noted, including an assessment of its grade of severity according to the United Nations World Health Organization (WHO) Toxicity Grading Scale. Twelve months after initiation of IPT, an additional retrospective medical record review was performed to document treatment completion.

While alternative therapies for prevention of TB reactivation were also prescribed at our health center, including 4-month courses of rifampicin, this report presents data relating to the first 100 patients prescribed the most common regimen of a 9-month course of isoniazid therapy (93\% of all prescriptions over the reported period; data not presented).

Standard counselling for IPT was provided to all patients prior to commencement. This included discussions of well-established potential adverse effects (particularly hepatitis, peripheral neuropathy, and allergic reactions); encouraging patients to report any potentially adverse effects; and provision of an information sheet about LTBI and its treatment.

Treatment completion for isoniazid was defined as receipt of the planned 9-month course within a 12-month period. It was pre-specified that descriptive analysis of outcomes would be conducted following the enrollment of 100 patients. This sample size was calculated to have more than $80 \%$ power (alpha $=0.05$ ) to detect side effects occurring with a frequency of 0.02 or greater in our cohort. Statistical calculations were performed in Microsoft Excel and StataCorp Stata 10. Fisher's exact test or chi-squared tests with two-tailed $P$-values were performed for betweengroup comparisons of dichotomous categories. In comparing continuous variables, $t$-tests were used.

\section{Results}

Demographic characteristics of the first 100 patients commenced on LTBI therapy are included in Table 1. The majority of patients were aged less than 35 years old, but 12 patients were aged 35-50 years old and nine were aged over 50 years old. Twelve patients had abnormalities of liver function at initiation of therapy, including six with hepatitis $B$ virus (HBV) infection.

Fifty-six patients reported at least one adverse effect at some stage during treatment. The number of patients reporting various adverse effects on at least one visit is presented in Table 2. The majority of adverse effects were Grade 1-2, with six patients experiencing at least one Grade 3-4 adverse effect

Table I Demographic characteristics of study cohort

\begin{tabular}{ll}
\hline Factor & Number \\
\hline Male & 58 \\
Age (median, range), years & 29 (16-72) \\
Region of birth & \\
Indian subcontinent & 38 \\
Asia-Pacific (excluding Australia) & 36 \\
Australia & 14 \\
Africa & 7 \\
Europe & 4 \\
South America & 1 \\
Comorbidities & \\
Hepatitis B & 6 \\
Hepatitis C & 0 \\
HIV & 1 \\
Liver disease (non-hepatitis) & 6 \\
Diabetes & 6 \\
Renal disease & 3 \\
\hline
\end{tabular}

Abbreviation: HIV, human immunodeficiency virus. 
Table 2 Adverse effects of isoniazid preventative therapy for latent tuberculosis infection

\begin{tabular}{lll}
\hline Adverse effect & $\begin{array}{l}\text { Number of } \\
\text { patients }\end{array}$ & $\begin{array}{l}\text { Grade 3-4 } \\
\text { severity }\end{array}$ \\
\hline Any adverse effect & 56 & 6 \\
Any gastrointestinal & 21 & 2 \\
Hepatitis & 5 & 1 \\
Any dermatologic & 15 & 1 \\
Any rash & 15 & 1 \\
Acne & 8 & 1 \\
Alopecia & 0 & 0 \\
Any neuropsychiatric & 19 & 1 \\
Lethargy & 7 & 0 \\
Cognitive impairment & 9 & 0 \\
Peripheral neuropathy & 4 & 0 \\
Headache & 2 & 0 \\
Sleep disturbance & 1 & 0 \\
Depression & 1 & 0 \\
\hline
\end{tabular}

during therapy. Increased age was significantly associated with risk of adverse effects (odds ratio $[\mathrm{OR}]=1.05$ per year; confidence interval $[\mathrm{CI}]$ of $1.02-1.08=95 \%$ ). Sex was not associated with risk of adverse effects; neither were any comorbid factors such as HBV infection or pre-existing liver or renal disease. Despite this frequency of adverse events, the majority of patients with adverse effects successfully continued therapy, with 46/56 (82\%) completing the planned course of treatment.

Of the first 100 patients started on 9-month courses of isoniazid, 85 patients successfully completed therapy in our health center. Three patients were transferred interstate, with documented arrangements to complete therapy. Twelve patients discontinued therapy, with ten citing adverse effects as the reason for treatment cessation and two patients failing to return for follow up. No hospitalizations or deaths occurred during the period of study. Of the 15 patients who did not complete treatment, no association with sex or age was found $(P=0.51$ and 0.78 , respectively).

\section{Gastrointestinal and hepatitic adverse effects}

The most common adverse effects overall were gastrointestinal, reported by 21 patients at some time during their course. Gastrointestinal symptoms tended to be identified at the initiation of treatment and spontaneously resolve; however, two patients discontinued therapy because of persistent gastrointestinal symptoms.

The mean age of the five patients who developed hepatotoxicity was 35.2 years old, while the mean age of the 95 patients who did not develop hepatotoxicity was 31.7 years (not significant). Only one patient experienced a Grade 3 or
4 hepatitis, with an alanine transferase (ALT) peak of 1,268 IU/L and bilirubin of $100 \mu \mathrm{mol} / \mathrm{L}$ approximately 4 months after starting therapy. This patient did not have pre-existing liver function test abnormalities or comorbidities at baseline. Isoniazid was ceased, with complete resolution of liver test abnormalities. The other four patients successfully completed treatment without interruption of therapy.

\section{Dermatological adverse effects}

Dermatological manifestations occurred in 15 patients, with eight patients developing acneiform rashes and eight patients developing generalized rashes (one patient experienced both at different times during treatment). No patient reported alopecia. Interestingly, while age was not a predictor of overall risk of rash, when grouped as "acneiform" and "non-acneiform" this effect was seen, with $8 / 8$ patients under 35 years of age experiencing acne. One patient with preexisting acne developed a severe flare, for which isotretinoin was prescribed after failure of topical therapy.

\section{Neuropsychiatric adverse effects}

The most common neuropsychiatric adverse effects reported were perceived cognitive impairment (nine patients) and lethargy (seven patients). A small number of patients reported headaches (two patients), sleep disturbance (one patient) and blurred vision (one patient). Four patients reported Grade 1 peripheral neuropathy, all relating to "pins and needles" and subjective sensory loss. One patient (of four) was receiving pyridoxine supplementation at the time of reporting symptoms. Three episodes were noted to occur late in therapy, with isoniazid discontinued as planned shortly thereafter. One episode occurred early in therapy, which resolved following introduction of pyridoxine. No association between peripheral neuropathy and sex or age was observed.

One patient experienced a transient psychosis 1 month after initiating isoniazid. The episode, marked by paranoia and hallucination, lasted approximately 1 week before spontaneously resolving, and was not reported until a subsequent clinic visit 2 months later. After discussion with the patient, treatment was continued without further incident.

\section{Discussion}

This report highlights a variety of somatic adverse effects that occurred in a real-world cohort of patients receiving IPT. While adverse effects were frequently identified in this study, the considerable majority of patients experienced low grade and transient effects. Importantly, despite observed adverse 
effects, this study has also demonstrated high levels of treatment adherence and completion in our cohort.

Prospectively-collected data on adverse effects and treatment completion are important for ensuring that patients are reasonably informed regarding the risks and benefits of isoniazid therapy. This is particularly the case given the increased range of age and comorbidities in real-world cohorts, as compared with those studied in many controlled trials. ${ }^{9}$

Variable (and frequently low) treatment completion rates have been reported in other cohorts receiving IPT, leading to concerns about the effectiveness of treatment as a preventative strategy. ${ }^{10,11}$ The findings from our cohort are reassuring in this regard, with $85 \%$ successful treatment completion demonstrated. This may reflect differences in our cohort, such as increased motivation due to perceived or actual high risk of contracting tuberculosis, or potential factors relating to routine pre-treatment counselling and follow up. Effective strategies for supporting good adherence are important, and further research toward such approaches would be valuable.

Five patients were observed to have abnormalities of liver function testing during therapy. While higher frequency of hepatotoxicity is recognized in older patients receiving isoniazid, we would observe that 4/5 patients in our cohort experienced transient mild elevations which resolved without cessation of therapy. ${ }^{12}$

In some settings, pyridoxine (PN) supplementation is given routinely for prevention of PN deficiency during IPT; however, the benefit of doing so for patients not at high risk is poorly established. In our cohort, $75 \%$ of those who reported symptoms of PN deficiency were not on pyridoxine. No definitive diagnostic test for isoniazid-associated PN deficiency exists, and patients reporting any symptoms potentially associated with PN deficiency were included in our report; the $4 \%$ of patients with symptoms is therefore likely to overestimate the risk.

Among adverse effects reported by patients, the frequency of lethargy and cognitive impairment was surprising, affecting 7 and 9 patients, respectively. While these symptoms tended to be mild, they were noticeable for patients. Information regarding adverse cognitive effects had not previously formed part of our routine counselling prior to this study, thus future guidelines will reflect increased recognition of these symptoms.
As IPT is used more widely and for increasingly broad indications, treatment guidelines should include realistic information on the frequency and severity of potential adverse effects. This information should be used to guide individuals and prescribing clinicians in making rational decisions about the risks and benefits of therapy, and should also include realistic plans for dealing with adverse effects, such as symptomatic strategies and alternatives for those unable to tolerate therapy. Overall, however, our experience has been that the majority of adverse effects are mild and transient, even in cohorts of older patients with comorbidities.

\section{Acknowledgment}

We thank the John Burge Trust Fund for financial support towards latent tuberculosis research during the period of this review.

\section{Disclosure}

The authors report no conflicts of interest in this work.

\section{References}

1. Denholm JT, McBryde ES. The use of anti-tuberculosis therapy for latent TB infection. Infect Drug Resist. 2010;3:63-72.

2. Zwerling A, van den Hof S, Scholten J, Cobelens F, Menzies D, Pai M. Interferon-gamma release assays for tuberculosis screening of healthcare workers: a systematic review. Thorax. 2012;67(1):62-70.

3. Fountain FF, Tolley E, Chrisman CR, Self TH. Isoniazid hepatotoxicity associated with treatment of latent tuberculosis infection: a 7-year evaluation from a public health tuberculosis clinic. Chest. 2005;128(1): 116-123.

4. Siskind MS, Thienemann D, Kirlin L. Isoniazid-induced neurotoxicity in chronic dialysis patients: report of three cases and a review of the literature. Nephron. 1993;64(2):303-306.

5. Gupta KB, Kumar V, Vishvkarma S, Shandily R. Isoniazid-induced alopecia. Lung India. 2011;28(1):60-61.

6. Dasta J, Prior J, Kurzrok S. Isoniazid-induced fever. Chest. 1979;75(2): 196-197.

7. Mann B. Isoniazid allergy. Br Med J. 1957;1(5020):685-686.

8. Street A, McBryde ES, Denholm JT, Eisen DP, editors. Management of Tuberculosis. 1st ed. Melbourne: Victorian Infectious Diseases Service; 2012.

9. Grapow MT, von Wattenwyl R, Guller U, Beyersdorf F, Zerkowski HR. Randomized controlled trials do not reflect reality: real-world analyses are critical for treatment guidelines! J Thorac Cardiovasc Surg. 2006;132(1):5-7.

10. Hirsch-Moverman Y, Daftary A, Franks J, Colson PW. Adherence to treatment for latent tuberculosis infection: systematic review of studies in the US and Canada. Int J Tuberc Lung Dis. 2008;12(11):1235-1254.

11. Kan B, Kalin M, Bruchfeld J. Completing treatment for latent tuberculosis: patient background matters. Int J Tuberc Lung Dis. 2013; 17(5):597-602.

12. Saukkonen JJ, Cohn DL, Jasmer RM, et al. An official ATS statement: hepatotoxicity of antituberculosis therapy. Am J Respir Crit Care Med. 2006;174(8):935-952. 
Drug, Healthcare and Patient Safety

Dovepress

\section{Publish your work in this journal}

Drug, Healthcare and Patient Safety is an international, peer-reviewed open-access journal exploring patient safety issues in the healthcare continuum from diagnostic and screening interventions through to treatment, drug therapy and surgery. The journal is characterized by the rapid reporting of reviews, original research, clinical, epidemiological and

post-marketing surveillance studies, risk management, health literacy and educational programs across all areas of healthcare delivery. The manuscript management system is completely online and includes a very quick and fair peer-review system. Visit http://www.dovepress.com/ testimonials.php to read real quotes from published authors.

Submit your manuscript here: http://www.dovepress.com/drug-healthcare-and-patient-safety-journal 